\title{
A coded-aperture technique allowing $x$-ray phase contrast imaging with conventional sources
}

\author{
Alessandro Olivo ${ }^{\text {a) }}$ and Robert Speller \\ Department of Medical Physics and Bioengineering, UCL, London WC1E 6BT, United Kingdom
}

(Received 1 May 2007; accepted 25 July 2007; published online 16 August 2007)

\begin{abstract}
Phase contrast imaging (PCI) solves the basic limitation of x-ray imaging, i.e., poor image contrast resulting from small absorption differences. Up to now, it has been mostly limited to synchrotron radiation facilities, due to the stringent requirements on the x-ray source and detectors, and only one technique was shown to provide PCI images with conventional sources but with limits in practical implementation. The authors propose a different approach, based on coded apertures, which provides high PCI signals with conventional sources and detectors and imposes practically no applicability limits. They expect this method to cast the basis of a widespread diffusion of PCI.

(c) 2007 American Institute of Physics. [DOI: 10.1063/1.2772193]
\end{abstract}

There is wide agreement on $\mathrm{x}$-ray phase contrast imaging (PCI) having the potential of revolutionizing $\mathrm{X}$-ray imaging. According to some authors, it can create "the biggest change in medical imaging since the invention of computed tomography," whereas others have focused on its potential in nonmedical fields, ${ }^{2,3}$ and likewise reported similar enhancements. The main problem is that PCI is still generally restricted to synchrotron radiation (SR) facilities due to the stringent requirements on the source and detector. To date, only one technique was shown to provide significant PCI signals with conventional sources, ${ }^{4,5}$ but still its practical implementation is subject to limitations. We propose here a different approach, based on the use of coded apertures, ${ }^{6}$ which makes PCI feasible with most commercially available $\mathrm{X}$-ray sources and detectors while removing most limitations of the previous methods.

Amongst the different PCI techniques, early studies were mostly based on free-space propagation. ${ }^{7,8}$ This simple implementation translates into poor phase contrast when used outside SR facilities, unless the source is strictly microfocal. This translates into excessively long exposure times in most practical applications. The negative effect of excessive source size and limited detector resolution can be assessed by means of convolution integrals.

Another class of methods involves the use of perfect crystals. These either use crystals in the arrangement of an $\mathrm{x}$-ray interferometer ${ }^{10}$ or they use a single crystal in the socalled diffraction enhanced imaging (DEI) approach, where it is used to analyze the microdeviations in photon direction due to the phase shift generated by the sample. The name DEI was introduced by Chapman et al., ${ }^{11}$ although the approach was used before by other authors. ${ }^{12,13}$ Crystal approaches provide an excellent image quality over a wide range of samples, but their use outside SR is made extremely difficult as they necessitate parallel, monochromatic radiation.

The most promising technique developed for PCI with conventional sources is based on a shearing interferometer. The method was first described by Clauser ${ }^{4}$ and more recently by Pfeiffer et al..$^{5}$ The beam is sliced into arrays of

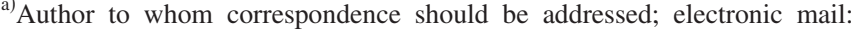
aolivo@medphys.ucl.ac.uk individually coherent but mutually incoherent beams by a grating placed close to the source. This allows PCI to be carried out with large source sizes, but it severely limits the angular divergence of the beam. The grating parallel apertures, longer along the x-ray propagation direction than in the transverse one, translate into a limited angular acceptance, and therefore in a substantial exposure time increase with nonparallel beams. The method accepts a maximum spectral bandwidth of about $10 \%$, whereas it was demonstrated that bandwidths of $50 \%$ or more can be used in PCI without affecting image quality, ${ }^{9}$ which would result in a more efficient use of the source. Two gratings are placed downstream of the sample, resulting in inefficient dose delivery, which is important in medical applications. Other limitations involve non-negligible sensitivity to vibrations due to small aperture dimensions $(\sim 2 \mu \mathrm{m})$, field of views currently limited to $6 \times 6 \mathrm{~cm}^{2}$, and sensitivity to phase effects in one-dimensional (1D) only [although Clauser suggests a method to achieve two-dimensional (2D) sensitivity].

We propose here an alternative method removing the above limitations. The idea originates from a SR experiment, in which it was demonstrated that the phase sensitivity of an

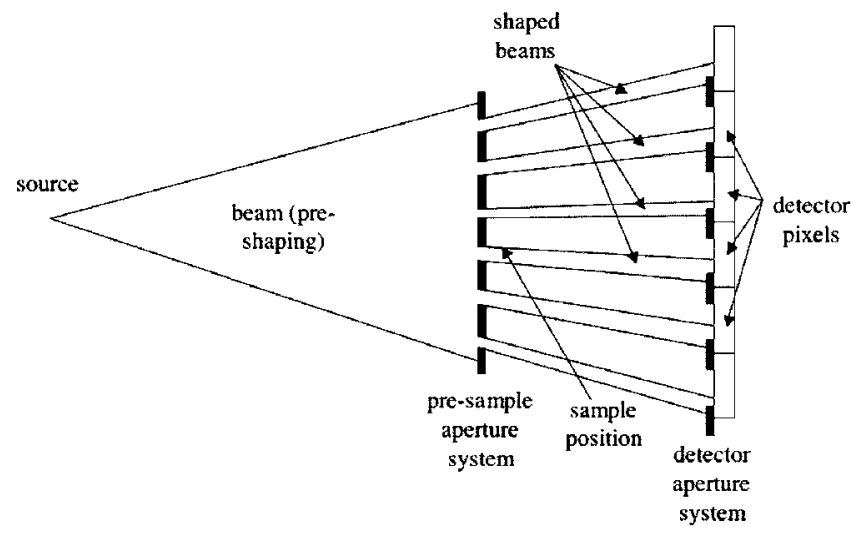

FIG. 1. Schematic of the experimental setup (not to scale). The beam created by the source is subdivided in a multiplicity of individual beams by the sample mask. Each of these beams impinges on the edge of the sensitive area of a detector pixel, as defined by the detector mask. The source to sample mask distance was $1.6 \mathrm{~m}$, sample mask to detector was $0.4 \mathrm{~m}$, the slit apertures in both masks were $50 \mu \mathrm{m}$ wide, and the slit pitches were 203 and $254 \mu \mathrm{m}$ in the sample and detector mask, respectively. 

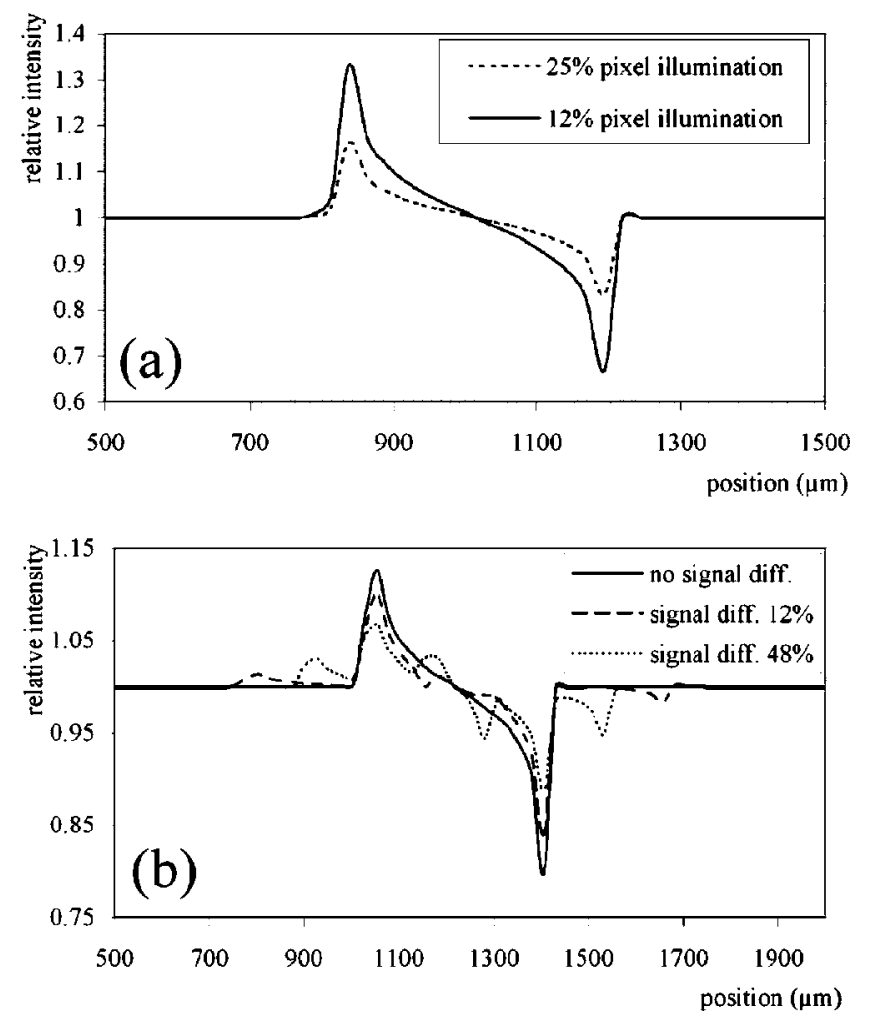

FIG. 2. Simulated profiles of a $300 \mu \mathrm{m}$ polyethylene fiber. (a) shows how the peak-to-peak signal (equivalent to the fiber contrast in the image) grows from $35 \%$ to $65 \%$ when the illuminated fraction of the pixel is reduced from $25 \%$ (dashed line) to $12 \%$ (solid line). (b) shows the same plot for $25 \%$ illumination without (solid line) and with 12\% (dashed line) and $48 \%$ (dotted line) signal diffusion between adjacent pixels. It can be seen that the effect of signal diffusion is an overall signal reduction and the appearance of parasite peaks, the intensity of which grows with increased signal diffusion. This parasite peaks would create additional interference fringes along the edges of the details in an image, without affecting their detectability; however, the influence of these extra fringes can be made negligible by the choice of the appropriate detector.

imaging system is dramatically enhanced by illuminating only the edge of the detector pixels. ${ }^{14}$ By doing so, the small deviations caused by phase shifts are sufficient to deflect outside the pixel surface, a photon that would have hit it in the absence of the sample. Likewise, a photon originally impinging immediately outside the pixel surface can be deviated enough to be detected. This explains the origin of the negative and positive peaks in the acquired signal. A situation similar to DEI, i.e., a fine selection of photon direction, is realized without using an analyzer crystal. In DEI, image contrast is increased by progressively rocking the analyzer crystal off the position corresponding to maximum reflection for the primary photons (Bragg angle). A similar situation is obtained here by reducing the illuminated fraction of the pixel.

In the SR experiment, the situation was made easier by the use of a parallel, laminar radiation beam and a detector consisting in a single row of pixels. The idea is transferred here to a laboratory environment, i.e., to a divergent, polychromatic beam and an area detector. This was obtained by designing an appropriate set of coded apertures, as shown in Fig. 1. The first set of coded apertures ("detector mask") creates insensitive regions between adjacent pixels. This mask was finely aligned with the detector pixels and then left in place throughout the experiment, but in a dedicated system, it could be laminated directly onto the detector surface. Downloaded 10 Sep 2008 to 144.82.107.152. Redistribution subje
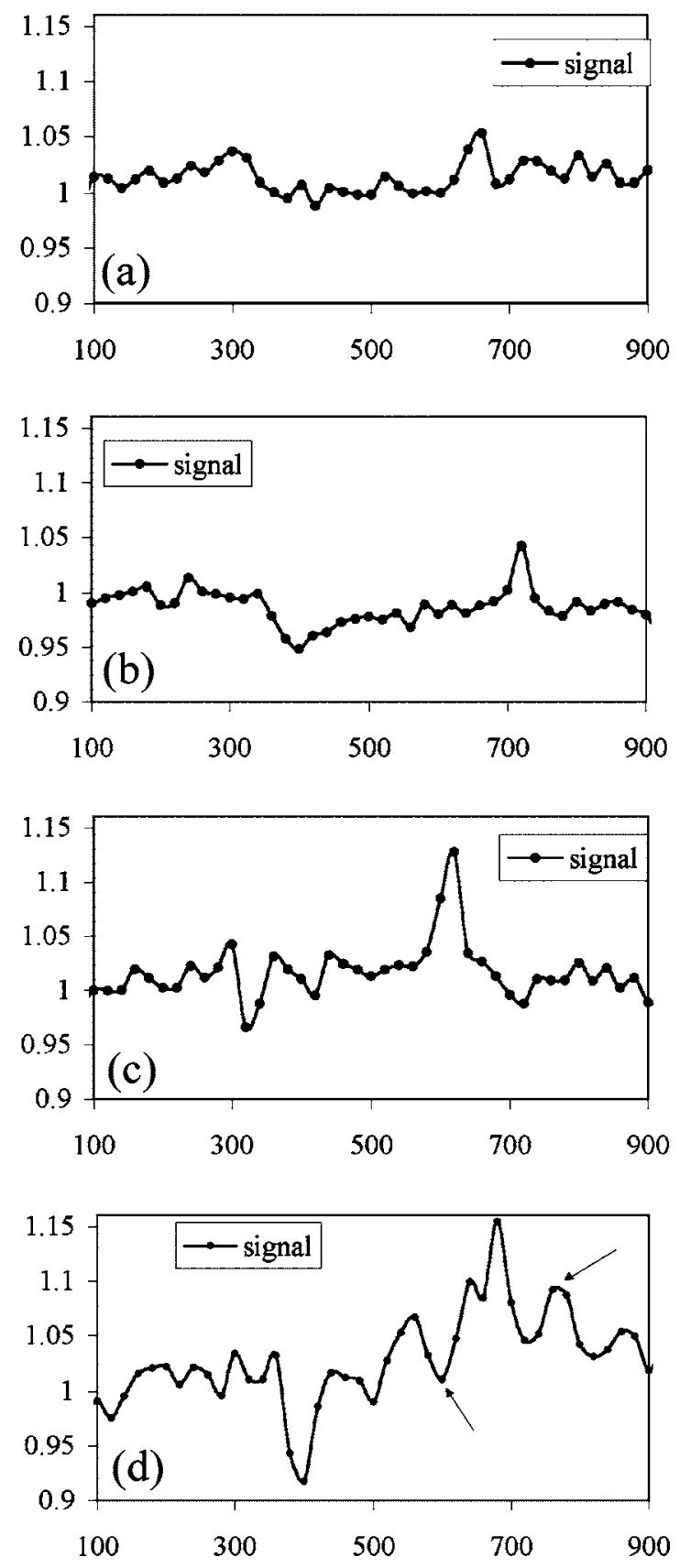

FIG. 3. Profiles extracted from experimentally acquired images of a $300 \mu \mathrm{m}$ polyethylene fiber. The illuminated fraction of the pixel was progressively reduced going from (a) to (d), which corresponds to a minimum illuminated fraction of about $25 \%$. As a consequence, the contrast is increased from $6 \%$ (a) to $9 \%$ (b), $16 \%$ (c), and $24 \%$ (d). Arrows in (d) underline the presence of parasite peaks due to the signal sharing effects. In all graphs, the relative intensity is given as a function of the spatial displacement in micron.

The second set of coded apertures ("sample mask") is placed immediately upstream of the sample, and its dimensions are scaled down according to the beam divergence. It creates a multiplicity of individual beams, each one hitting one of the sharp pixel edges created by the detector mask. This arrangement replaces the sample scanning applied with SR to obtain $2 \mathrm{D}$ images, meaning that no sample or mask scanning is required during image acquisition.

Assuming a 50\% fill factor for the sample mask, the method would require a twofold increase in exposure time with respect to a conventional system, which is a significant improvement with respect to other techniques in which this increase is at least of one order of magnitude. The masks can to AIP license or copyright; see http://apl.aip.org/apl/copyright.jsp 
be designed in such a fashion that the aperture edges are parallel to the local direction of the primary photons, meaning that no angular filtering is applied. Moreover, no chromatic filtering is required, and therefore the full spectrum can be exploited as previously suggested. ${ }^{9}$

Although for the present proof-of-principle experiment long apertures were carved in the masks, i.e., a system sensitive to the phase effects in 1D only was realized, the technique is easily extendable to $2 \mathrm{D}$ by designing L-shaped apertures matching two edges of each detector pixel.

A ray-tracing approach was used to simulate the system. Two relevant aspects were observed: the intensity of the PCI signal grows significantly by reducing the pixel fraction illuminated by $\mathrm{x}$ rays and the signal diffusion between adjacent pixels affects the intensity and the shape of the acquired signal. This signal diffusion can be due to different factors (charge sharing, crosstalk, etc.), although the most relevant one in most x-ray detectors is light diffusion in the scintillator. Direct-conversion systems, or at least structured scintillators, would thus be the detectors of choice for the technique. These effects are shown in Fig. 2, where profiles of a $300 \mu \mathrm{m}$ thick polyethylene fiber simulated under different conditions are shown.

To validate the model, images of such a fiber were acquired experimentally. A molybdenum target $\mathrm{x}$-ray source manufactured by X-Tek (X-Tek Systems Ltd., Tring, UK) was used. It features a $100 \mu \mathrm{m}$ focal spot, $45^{\circ}$ angular divergence and allows a power loading on the source of $375 \mathrm{MW} / \mathrm{cm}^{2}$. The Varian PaxScan 4030R flat panel imager (Varian, Salt Lake City, UT) was used as detector. It features an unstructured gadox screen, which makes it not ideal for the proposed technique, but the aim at this stage was just to obtain the proof of concept. For the same reason, sample and detector masks featuring only five long apertures were designed and manufactured. Larger fields of views were then obtained by repeating the exposure after moving the sample in steps and pasting the collected images together, although this resulted in an overall noise increase in the final image. The maximum [i.e., the one used to obtain Fig. 3(d) (see below)] exposure time per frame was of $3 \mathrm{~s}$, which has to be multiplied by the number of frames acquired (typically $\sim 20$ ) to obtain a full "pasted" image. The realization of a set of masks covering the entire detector surface (currently underway) will make the acquisition of a single frame sufficient, thus keeping the overall exposure times within $3 \mathrm{~s}$ or less.

Four (pasted) fiber images were obtained by progressively shifting the sample mask with respect to the detector one in order to illuminate gradually smaller fractions of the pixel (i.e., downward with respect to Fig. 1). The profiles extracted from these images are shown in Fig. 3. It is worth noting that the absorption signal for the same object would be of about $1 \%$, meaning that a 24 -fold contrast increase was obtained in the image corresponding to Fig. 3(d). The traces of parasite peaks due to signal sharing visible in the plots further confirm the simulation's prediction. It should be noted that higher signals would be obtained by utilizing a detector affected by less signal sharing and by further reducing the illuminated fraction of the pixel. This last point is very significant; the technique allows the user to amplify the contrast by trading off on the exposure time.

The sample mask prevents unnecessary dose delivery: the only source of extradose originates from the fraction of each beam impinging on the insensitive regions on the detector mask (which gives rise to the positive peaks in the signal thus doubling the contrast). However, this fraction can be kept equal to a small percentage $(10 \%-20 \%)$ of the individual beam cross section. Although the delivered dose was not evaluated in this case as the imaged sample was not a thick, biological one, this means that the dose increase required by the proposed system with respect to conventional ones is of the order of $10 \%-20 \%$ at the most.

In order to maintain the flexibility on the exposure time/ image contrast trade-off, obtained by shifting the sample mask with respect to the detector one, in those cases in which dose is an issue, a sample mask consisting in two superimposed masks should be realized. As one is pushed in one direction to reduce the pixel illumination, the other would be used to reduce the increased portion of the beam hitting the insensitive region on the detector mask, keeping it equal to $10 \%-20 \%$ of the total.

During the completion of this work, one of the authors (A.O.) was supported by a Marie Curie Intra-European Fellowship within the Sixth European Community Framework Programme, Contract No. MEIF-CT-2004-009143.

${ }^{1}$ R. Lewis, Phys. Med. Biol. 49, 3573 (2004).

${ }^{2}$ P. Cloetens, M. Pateyron-Salome, J. Y. Buffiere, G. Peix, J. Baruchel, F. Peyrin, and M. Schlenker, J. Appl. Phys. 81, 5878 (1997).

${ }^{3}$ Z. W. Hu, P. A. Thomas, A. Snigirev, I. Snigireva, A. Souvorov, P. G. R. Smith, G. W. Ross, and S. Teat, Nature (London) 392, 690 (1998).

${ }^{4}$ J. F. Clauser, U.S. Patent No. 5812629 (September 22, 1998).

${ }^{5}$ F. Pfeiffer, T. Weitkamp, O. Bunk, and C. David, Nat. Phys. 2, 258 (2006).

${ }^{6}$ A. Olivo and R. Speller, U.K. Patent Application GB0617637.4.

${ }^{7}$ A. Snigirev, I. Snigireva, V. Kohn, S. Kuznetsov, and I. Schelokov, Rev. Sci. Instrum. 66, 5486 (1995).

${ }^{8}$ S. W. Wilkins, T. E. Gureyev, D. Gao, A. Pogany, and A. W. Stevenson, Nature (London) 384, 335 (1996).

${ }^{9}$ A. Olivo and R. Speller, Phys. Med. Biol. 51, 3015 (2006).

${ }^{10}$ A. Momose, T. Takeda, Y. Itai, and K. Hirano, Nat. Med. 2, 473 (1996).

${ }^{11}$ D. Chapman, W. Thomlinson, R. E. Johnson, D. Washburn, E. Pisano, N. Gmur, Z. Zhong, R. Menk, F. Arfelli, and D. Sayers, Phys. Med. Biol. 42, 2015 (1997).

${ }^{12}$ V. N. Ingal and E. A. Beliaevskaya, J. Phys. D 28, 2314 (1995).

${ }^{13}$ T. J. Davis, D. Gao, T. E. Gureyev, A. W. Stevenson, and S. W. Wilkins, Nature (London) 373, 595 (1995).

${ }^{14}$ A. Olivo, F. Arfelli, G. Cantatore, R. Longo, R. H. Menk, S. Pani, M. Prest, P. Poropat, L. Rigon, G. Tromba, E. Vallazza, and E. Castelli, Med. Phys. 28, 1610 (2001). 